\title{
Adaptation transculturelle et validation française du questionnaire de sensibilité à la douleur (Pain Sensitivity Questionnaire)
}

\section{Transcultural adaptation and French validation of the Pain Sensitivity Questionnaire}

\author{
Christian Dualé, MD, PhD (i) - Ulrich Bauer, MB • Brigitte Storme, MB • Vedat Eljezi, MB, PhD • \\ Ruth Ruscheweyh, MD - Sylvie Eschalier, BN • Claude Dubray, MD, PhD • Candy Guiguet-Auclair, PhD
}

Received: 30 October 2018/Revised: 26 March 2019/Accepted: 27 March 2019/Published online: 24 April 2019

(C) Canadian Anesthesiologists' Society 2019

\section{Résumé}

Objectif Valider une version en langue française du Pain Sensitivity Questionnaire (PSQ), qui permet d'identifier la sensibilité naturelle d'un individu à la douleur, ce qui pourrait être applicable après une chirurgie.

Méthode Nous avons étudié la validité de structure interne, la validité convergente (anxiété, dépression et catastrophisme) et la reproductibilité par test-retest de la version française du PSQ (PSQ-F) chez 146 patients en situation préopératoire ou en cours de grossesse, puis la validité convergente et de structure contre critère externe chez 85 sujets volontaires sains soumis à des tests nociceptifs.

Résultats $\mathrm{La}$ consistance interne $d u \quad P S Q-F$ était excellente avec des $\alpha$ de Cronbach égaux à 0,866 , 0,886 et 0,927 , respectivement pour ses scores « mineur »,

Electronic supplementary material The online version of this article (https://doi.org/10.1007/s12630-019-01377-w) contains supplementary material, which is available to authorized users.

C. Dualé, MD, PhD $(\bowtie) \cdot C$. Dubray, MD, PhD

Centre de Pharmacologie Clinique (INSERM CIC1405), CHU

de Clermont-Ferrand, 58 rue Montalembert, BP 69, 63003

Clermont-Ferrand Cedex 1, France

e-mail: cduale@chu-clermontferrand.fr

INSERM, UMR1107 Neuro-Dol, Clermont-Ferrand, France

U. Bauer, MB

Centre Jean-Perrin, Anesthésie-Réanimation, Clermont-Ferrand, France

B. Storme, MB · V. Eljezi, MB, PhD

CHU Clermont-Ferrand, Médecine Péri-Opératoire, Clermont-

Ferrand, France «modéré » et «total». La reproductibilité était satisfaisante, avec des coefficients de corrélation intra-classe, respectivement à 0,629, 0,629 et 0,635. Ces trois scores étaient corrélés à l'anxiété, la dépression et le catastrophisme, mais pas chez les volontaires sains qui avaient des scores psychométriques bas et peu variables. Ils étaient anti-corrélés au seuil de nociception thermique chaud en épreuve supra-liminale, mais pas au seuil de nociception mécanique ponctuelle, ni au seuil de nociception thermique chaud en épreuve liminale. Enfin, les scores «mineur »et «total étaient corrélés à la douleur moyenne ressentie à l'immersion du pied en eau froide.

Conclusion Cette version validée peut être utilisée par les chercheurs et cliniciens francophones.

Enregistrement de l'étude $w w w$.ClinicalTrials.gov (NCT03113903); le 14 avril 2017.

R. Ruscheweyh, MD

Department of Neurology, University Hospital, LMU Munich, Munich, Germany

S. Eschalier, BN

Centre de Pharmacologie Clinique (INSERM CIC1405), CHU de Clermont-Ferrand, 58 rue Montalembert, BP 69, 63003 Clermont-Ferrand Cedex 1, France

C. Guiguet-Auclair, $\mathrm{PhD}$

CHU Clermont-Ferrand, Santé Publique, Clermont-Ferrand, France

CNRS, Institut Pascal, Clermont-Ferrand, France 


\begin{abstract}
Purpose To validate a French translation of the Pain Sensitivity Questionnaire (PSQ), which is a valuable tool to predict an individual's natural disposition to feel pain that could be used after surgery.
\end{abstract}

Methods We studied content validity, internal consistency, convergent validity (anxiety, depression and catastrophism) and test-retest reliability of the French version of the PSQ (PSQ-F) in 146 patients either before scheduled surgery or during pregnancy; then, convergent and concurrent validity in 85 healthy volunteers submitted to nociceptive tests.

Results Internal consistency of the PSQ-F was found to be excellent, with Cronbach's $\alpha$ at 0.866, 0.886, and 0.927, respectively for its "minor", "moderate" and "total" scores. Test-retest reliability was significant, with intraclass correlation coefficients at 0.629, 0.629, and 0.635 , respectively for the above- mentioned scores. These three scores correlated with anxiety, depression and catastrophizing scores in patients, but not in healthy volunteers, possibly because of low and few variant psychometric scores in this group. They were inversely correlated to the temperature needed to evoke heat pain rated 6 out of 10, but not to the mechanical pain threshold (electronic von Frey), nor to the heat pain threshold. Finally, they directly correlated to the pain induced by the cold pressor test (minor and total scores only).

Discussion This validated version can now be used by French-speaking researchers and physicians.

Trial registration www.ClinicalTrials.gov (NCT03113903); 14 April, 2017.

La prédisposition à la douleur repose sur des mécanismes complexes, et fait - par exemple - que pour un geste chirurgical identique, la douleur postopératoire varie d'un patient à l'autre. ${ }^{1,2}$ Or, il semble important de pouvoir prédire la douleur postopératoire pour anticiper l'analgésie à appliquer. Dans le contexte obstétrical, personnaliser l'analgésie du travail permettrait en sus d'éviter des excès d'analgésie, autre motif d'insatisfaction de la patiente. ${ }^{3} \mathrm{On}$ peut déjà prédire la douleur postopératoire/obstétricale ou les besoins en analgésie par la sensibilité préopératoire à une stimulation nociceptive standardisée, ${ }^{4-8}$ mais de telles mesures sont difficiles à réaliser en pratique et ajoutent une épreuve douloureuse au patient. Le Pain Sensitivity Questionnaire (PSQ) explore la sensibilité naturelle d'un individu à la douleur au moyen de questions simulant des situations douloureuses plus ou moins intenses de la vie courante. ${ }^{9}$ Il pourrait être utile en recherche clinique (stratification lors d'essais d'analgésiques, physiopathologie...) mais aussi en clinique courante, intégré à la construction de scores de risque de douleur postopératoire. ${ }^{10}$ Originellement construit en langue allemande, le PSQ a fait l'objet de traductions validées, ${ }^{11-14}$ mais une validation transculturelle en langue française est nécessaire. C'est l'objectif de cette étude, qui veut étudier en deux étapes : (i) la validité de structure interne (degré de corrélation entre les items et au sein de dimensions), la validité convergente (corrélations avec d'autres mesures du même domaine) et la reproductibilité (stabilité des réponses d'un sujet dont l'état est resté stable au cours du temps) chez des patients en situation préopératoire ou des femmes enceintes avant accouchement; (ii) la validité convergente et la validité de structure contre critère externe (corrélations avec des indicateurs objectifs de sensibilité à la douleur) chez des sujets volontaires sains.

\section{Méthode}

Le plan est conforme aux recommandations pour la validation transculturelle, comportant une traduction littérale, une adaptation au contexte culturel, aux habitudes de vie et aux idiomes de la population cible, et suivant un processus rigoureux de traduction-contre-traduction. ${ }^{15-17}$ Cette étude a reçu l'autorisation de l'Agence Nationale de Sécurité du Médicament et des produits de santé (autorité compétente) le 10/04/2017, et l'avis favorable du comité d'éthique (CPP-Ile-de-France-I) le 08/04/2017. Elle a été enregistrée sur ClinicalTrials.gov (NCT03113903). Tous les participants ont donné leur consentement éclairé préalable.

La première partie incluait des patients du $\mathrm{CHU}$ de Clermont-Ferrand et du Centre Jean-Perrin, âgés de 18 à 65 ans, parlant et lisant le français, à l'occasion de la visite réglementaire pré-anesthésique, qui se déroulait environ deux semaines avant une chirurgie programmée, ou au troisième trimestre de la grossesse. Tous les patients éligibles ont été sollicités de façon consécutive au cours de la période d'étude. Après la collecte des données démographiques et morphologiques, et des antécédents du patient, ce dernier était invité à renseigner à la suite : le PSQ-F, l'échelle de catastrophisme vis-à-vis de la douleur de Sullivan et coll. (Pain Catastrophizing Scale, PCS), ${ }^{18,19}$ et l'échelle d'anxiété et de dépression en hôpital (Hospital Anxiety \& Depression, HADS), ${ }^{20,21}$ toutes deux validées en langue française. Si possible, les patients étaient revus peu avant l'intervention pour renseigner une seconde fois le PSQ-F afin d'évaluer sa reproductibilité par test-retest, puis contactés entre le deuxième et le troisième jour après l'intervention (ou l'accouchement), pour répondre à la question «D'une façon générale, quelle est votre 
satisfaction vis-à-vis de la prise en charge de la douleur par rapport à votre opération ou votre accouchement ? » sur une échelle numérique simple allant de 0 ( « Pas du tout satisfait ») à 10 (« Totalement satisfait »).

La seconde partie incluait des sujets volontaires sains recrutés par téléphone au Centre de Pharmacologie Clinique à partir de la base de participants du centre. Ils étaient âgés de 18 à 60 ans, ayant un indice de masse corporelle entre 19 et $30 \mathrm{~kg} \cdot \mathrm{m}^{-2}$, indemnes de tout traitement dans les sept jours précédant l'étude, parlant et lisant le français. Le recrutement a visé une distribution équilibrée entre les sexes et de part et d'autre d'un âge de 30 ans. Les critères de non-inclusion étaient : grossesse, incompatibilité cognitive avec l'étude, alcoolisme avéré, addiction aux drogues, douleur chronique, tout antécédent médical ou toute pathologie contre-indiquant la réalisation de l'étude (notamment : lésion cutanée dans les régions à explorer, score d'anxiété ou de dépression $>11$ au questionnaire HADS, qui de fait était renseigné et interprété avant l'inclusion formelle). Tous les tests ont été réalisés en une fois à l'occasion de la visite au Centre. Un test urinaire de dépistage de substances addictives (NarcoCheck ${ }^{\circledR}$, Kappa City Biotech SAS, Montluçon, France) a été systématiquement réalisé. Les questionnaires ont été renseignés dans un lieu dédié, confortable et silencieux, sans influence des investigateurs.

Les mesures psychophysiques ont été réalisées en environnement calme, à température fixe de $24^{\circ} \mathrm{C}$. Après un court apprentissage, le seuil de sensibilité à la douleur mécanique ponctuelle a été mesuré au moyen du von Frey électronique (Somedic, Hornby, Suède) sur l'avant-bras en distal. Puis, au moyen du dispositif Pathway ${ }^{\circledR}$ (Medoc Ltd, Ramat Yishai, Israël), les seuils de sensibilité et de douleur induite par un stimulus chaud ont été mesurés sur le même avant-bras en proximal. A partir de la mesure du seuil de douleur au chaud, a été ensuite mesuré le seuil supra-liminal « T\%/douleur-6 », correspondant à l'obtention d'une douleur équivalente à $6 / 10$ sur une échelle verbale numérique. Enfin, la sensibilité à la douleur au froid a été explorée par une épreuve d'immersion du pied non dominant dans un bain d'eau à $4^{\circ} \mathrm{C}$ pendant deux minutes (cold pressure test, CPT); les critères de jugement étaient la douleur moyenne ressentie, soit pendant les deux minutes entières, soit pendant les 30 premières secondes. Le seuil de 30 secondes étant la médiane observée pour le sous-groupe des sujets ayant retiré le pied avant la fin de la période de deux minutes, cette mesure intermédiaire est indépendante de la tolérance sur la durée. Le détail du protocole est fourni sous eAnnexe (disponible sous le Matériel électronique supplémentaire).

Afin de tester les propriétés psychométriques du PSQ-F (acceptabilité, validité de structure interne, reproductibilité par test-retest, validité convergente et validité de structure contre critère externe), ont été retenues comme variables dépendantes les items du questionnaire PSQ, et - sous réserve de validité de la structure interne - le score total du PSQ (moyenne des scores hors items n ${ }^{\circ} 5-9-13$, qui sont des leurres ne représentant pas la douleur), le sous-score «mineur» du PSQ (moyenne des scores sur items $\mathrm{n}^{\circ}$ 3-6-7-10-11-12-14), et le sous-score «modéré » du PSQ (moyenne des scores sur items $\left.\mathrm{n}^{\circ} 1-2-4-8-15-16-17\right)$. Les items composant les sous-scores « mineur » et «modéré » correspondent - sur une échelle de 0 à 10 - à des situations génératrices de douleur cotées respectivement inférieure à 4 , et entre 4 et $6{ }^{9}$ Les facteurs étudiés dans les études de relation étaient : les scores d'anxiété, de dépression et de catastrophisme ainsi que ses trois sous-scores (amplification, rumination et abandon), les seuils de nociception mécanique ponctuelle et thermique chaud (basal et $\mathrm{T} \%$ douleur-6), et le délai de retrait et les moyennes d'intensité de douleur (sur 30 secondes et 120 secondes) au CPT. Le contexte chirurgical a été pris en compte pour analyses en sous-groupes (de préférence au sexe et à l'âge, naturellement imbriqués avec cette variable).

Les données qualitatives ont été présentées en termes d'effectifs et pourcentages, et les données quantitatives en termes de moyenne (écart-type) ou médiane (écart inter-quartile). Les tests statistiques étaient bilatéraux avec un seuil de significativité fixé à $5 \%$. La mesure de l'acceptabilité a reposé sur le calcul des données manquantes au niveau de chaque item du PSQ. Des taux de remplissage complet du PSQ-F et des taux de réponse à chacun des 17 items supérieurs à $85 \%$ étaient considérés comme satisfaisants. La présence d'effet plafond ou plancher a été recherchée. Afin de déterminer si les items du PSQ-F étaient discriminants, le pourcentage de sujets ayant le score le plus élevé (effet plafond) et le pourcentage de sujets ayant le score le plus faible (effet plancher) ont été calculés pour chaque score (total, mineur et modéré). La validité de structure interne a été appréciée par une analyse factorielle avec rotation Varimax. La consistance interne de chacun des facteurs identifiés a été évaluée par le coefficient $\alpha$ de Cronbach. Les corrélations inter-items, items-facteurs et inter-facteurs ont été estimées par des coefficients de corrélation de Pearson ou Spearman au regard de la distribution statistique. La reproductibilité a été appréciée par des coefficients de corrélation intra-classe. La validité convergente a été étudiée en calculant les coefficients de corrélation de Pearson ou Spearman au regard de la distribution statistique, entre les scores du PSQ-F et les scores des échelles HADS et PCS. La validité de structure contre critère externe a été étudiée en calculant les coefficients de corrélation entre les scores du PSQ-F et les scores issus des différentes stimulations nociceptives standardisées. En raison de la nature exploratoire des comparaisons statistiques, aucun 
ajustement pour comparaison multiple n'a été effectué. ${ }^{22,23}$ Pour les analyses des effets de facteurs confondants démographiques ou nosologiques, des coefficients de corrélation avec les variables numériques ont été calculés, et des comparaisons entre sous-groupes ont été réalisées par des tests de Student ou de Mann-Whitney.

Pour les patients, nous avons déterminé le nombre minimum de sujets nécessaires à 136 sujets en fonction du nombre d'items du PSQ-F (huit sujets/item, 17 items), pour une validité raisonnable de l'analyse factorielle. ${ }^{24} \mathrm{Ceci}$ permet de détecter des coefficients de corrélation $\geq 0,3$ avec $\alpha=5 \%$ et $1-\beta=95 \%$. L'objectif de recrutement était porté à 150 par sécurité. Par ailleurs, 50 patients étaient nécessaires pour étudier la reproductibilité. ${ }^{24}$ Pour les volontaires sains, nous avons fait l'hypothèse que les corrélations entre les scores du PSQ-F et les variables psychophysiques seraient au moins aussi importantes que celles retrouvées dans l'étude originale ( $\rho$ entre 0,29 et 0,59). ${ }^{9}$ Pour détecter des coefficients de corrélation $\geq 0,3$ avec $\alpha=5 \%$ et $1-\beta=80 \%, 85$ sujets étaient nécessaires.

\section{Résultats}

Traduction et adaptation transculturelle

Nous sommes partis de la version originale allemande du PSQ, ${ }^{9}$ qui a été traduite séparément de l'allemand au français par trois bilingues de langue maternelle française. Une traduction à partir de la version anglaise a été aussi réalisée en parallèle. Puis une version synthétique a été rédigée, et rétro-traduite par deux bilingues de langue maternelle allemande natifs ignorants du PSQ original. Ces rétro-traductions ont été comparées à l'original, puis une version pré-finale a été produite et testée auprès d'un échantillon de 40 personnes soignantes en milieu hospitalier. Ces personnes ont estimé le temps qu'elles ont pris pour remplir le questionnaire, évalué la compréhensibilité des consignes de lecture et des questions/mots, et noté le recours éventuel à une aide extérieure; elles ont pu faire des suggestions de correction ou d'ajout d'items. Le temps moyen passé à remplir était de cinq minutes, sans avoir recours à une aide. Suite à ce test, seule une consigne de remplissage a été modifiée (« face à une telle situation » a été remplacée par « face à la situation évoquée »). Aucun item n'a été modifié. Une version finale (PSQ-F) a ainsi été adoptée (Tableau 1).

Description des patients et des sujets sains

Le diagramme des flux est détaillé en Figure 1. Les caractéristiques des participants sont au Tableau 2. Chez les patients, l'âge présentait une distribution bimodale avec deux modes à 30 et 60 ans. Les types de chirurgie programmée étaient : abdomen/pelvis 8 (5,5\%), cervical $13(8,9 \%)$, cœur $20(13,7 \%)$, obstétrique $38(26,0 \%)$, orthopédie $11(7,5 \%)$, sein $36(24,7 \%)$, thorax $16(11,0$ $\%)$, autre $4(2,7 \%)$. Chez les sujets sains, la distribution était asymétrique du fait d'une différence de variabilité entre les moins et plus de 30 ans; le score global moyen de catastrophisme était de 11,6 (8,3), soit inférieur à celui l'étude originale, égal à $16,5(9,0)$ (données non publiées). ${ }^{9}$

Acceptabilité, effets planchers et plafonds

Le taux de remplissage complet du PSQ a été de $100 \%$ pour les deux groupes. Pour les 14 items correspondant à des situations douloureuses, les taux de réponses au niveau le plus bas ( 0 , effet plancher) allaient de 0 à $18,5 \%$ et de 0 à $15,3 \%$, respectivement pour les patients et les sujets sains. Les taux de réponses au niveau le plus haut (10, effet plafond) allaient de 0 à $6,2 \%$ et de 0 à $2,4 \%$, respectivement pour les patients et les sujets sains.

\section{Validité de structure interne et reproductibilité}

Le Tableau 3 montre les résultats de l'analyse factorielle du PSQ-F. Pour les patients, deux facteurs ont été retenus, représentant respectivement 51,7 et $8,4 \%$ de la variance totale. Le coefficient $\alpha$ de Cronbach estimant la consistance interne (considérée comme acceptable à partir de 0,7 ) était, respectivement pour les sous-score «mineur» et «modéré » et le score total du PSQ-F : 0,866; 0,886; 0,927 . Pour l'analyse de reproductibilité (test/retest), le coefficient de corrélation intra-classe était, respectivement pour les sous-score «mineur » et «modéré » et le score total du PSQ-F : 0,629; 0,629; 0,635. La corrélation intra-classe était significative pour tous les items et scores testés. Pour les sujets sains, deux facteurs ont également été retenus dans l'analyse factorielle, représentant respectivement 46,1 et $11,3 \%$ de la variance totale. Le coefficient $\alpha$ était, respectivement pour les sous-score «mineur » et «modéré » et le score total du PSQ-F : 0,852; 0,871;0,906.

Les composantes des sous-scores du PSQ original étant retrouvées comme attendues dans les dimensions de l'analyse factorielle, et la consistance interne de l'outil étant jugée satisfaisante, il n'a pas été procédé à des modifications du questionnaire ni des méthodes de calcul des scores, pour les analyses suivantes. On peut donc décrire les scores du PSQ-F (Tableau 4). On constate des scores plus élevés chez les sujets sains. Du fait de l'hétérogénéité des types de chirurgie, il n'a pas été réalisé d'analyse sur ces sous-groupes. 
Tableau 1 Version française du Pain Sensitivity Questionnaire (PSQ-F)

\section{Questionnaire de Sensibilité à la Douleur}

Ce questionnaire contient une série de questions pour lesquelles vous devez vous imaginer certaines situations. Vous devez alors décider si ces situations seraient douloureuses pour vous, et si c'est le cas, à quel point elles seraient douloureuses. La valeur 0 signifie « aucune douleur », 1 une douleur à peine perceptible, et 10 la pire douleur que vous puissiez imaginer ou penser possible.

Veuillez, s'il vous plait, entourer le chiffre qui vous semble le plus approprié. Il n'y a pas de réponse "juste» ou "fausse ». Il s'agit seulement de votre évaluation personnelle de la situation. Essayez si possible de ne pas intégrer dans l'évaluation de la douleur, la peur ou la crainte que vous pourriez ressentir face à la situation évoquée.

1. Imaginez que vous vous cognez fortement le tibia contre un bord dur, par exemple sur le bord d'une table basse en verre. À quel point ceci serait douloureux pour vous ? $0=$ Aucune douleur La plus forte douleur imaginable $=10$

\begin{tabular}{l|l|l|l|l|l|l|l|l|l|l}
0 & 1 & 2 & 3 & 4 & 5 & 6 & 7 & 8 & 9 & 10
\end{tabular}

\section{Imaginez que vous vous brûlez la langue avec une boisson très chaude.}

$0=$ Aucune douleur La plus forte douleur imaginable $=10$

\begin{tabular}{|l|l|l|l|l|l|l|l|l|l|l|}
\hline 0 & 1 & 2 & 3 & 4 & 5 & 6 & 7 & 8 & 9 & 10 \\
\hline
\end{tabular}

3. Imaginez que vous avez des douleurs musculaires après une activité physique (courbatures).

$0=$ Aucune douleur

La plus forte douleur imaginable $=10$

\begin{tabular}{|l|l|l|l|l|l|l|l|l|l|l|l|}
\hline 0 & 1 & 2 & 3 & 4 & 5 & 6 & 7 & 8 & 9 & 10 \\
\hline
\end{tabular}

\section{Imaginez que vous vous coincez un doigt dans un tiroir.}

$0=$ Aucune douleur

La plus forte douleur imaginable $=10$

\begin{tabular}{|l|l|l|l|l|l|l|l|l|l|l|}
\hline 0 & 1 & 2 & 3 & 4 & 5 & 6 & 7 & 8 & 9 & 10 \\
\hline
\end{tabular}

5. Imaginez que vous prenez une douche à l'eau tiède.

$0=$ Aucune douleur La plus forte douleur imaginable $=10$

\begin{tabular}{|l|l|l|l|l|l|l|l|l|l|l|l|}
\hline 0 & 1 & 2 & 3 & 4 & 5 & 6 & 7 & 8 & 9 & 10 \\
\hline
\end{tabular}

\section{Imaginez que vous avez un léger coup de soleil sur les épaules.}

$0=$ Aucune douleur

La plus forte douleur imaginable $=10$

\begin{tabular}{|l|l|l|l|l|l|l|l|l|l|l|}
\hline 0 & 1 & 2 & 3 & 4 & 5 & 6 & 7 & 8 & 9 & 10 \\
\hline
\end{tabular}

\section{Imaginez que vous vous écorchez le genou en tombant de vélo.}

$0=$ Aucune douleur

La plus forte douleur imaginable $=10$

\begin{tabular}{|l|l|l|l|l|l|l|l|l|l|l|}
\hline 0 & 1 & 2 & 3 & 4 & 5 & 6 & 7 & 8 & 9 & 10 \\
\hline
\end{tabular}

\section{Imaginez qu'en mangeant, vous vous mordez fortement la langue ou la joue par} accident. 
Tableau 1 continued

O=Aucune douleur

La plus forte douleur imaginable $=10$

\begin{tabular}{|l|l|l|l|l|l|l|l|l|l|}
\hline 0 & 1 & 2 & 3 & 4 & 5 & 6 & 7 & 8 & 9
\end{tabular}

9. Imaginez que vous marchez pieds nus sur un carrelage frais.

$0=$ Aucune douleur

La plus forte douleur imaginable $=10$

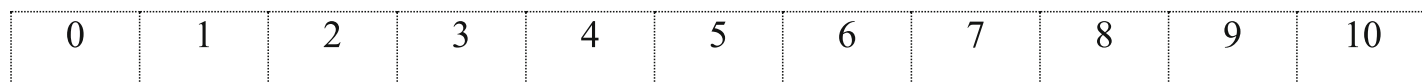

10. Imaginez que vous avez une petite blessure au doigt, et que, sans le faire exprès, vous vous mettez du jus de citron sur la plaie.

$0=$ Aucune douleur

La plus forte douleur imaginable $=10$

\begin{tabular}{|l|l|l|l|l|l|l|l|l|l|}
\hline 0 & 1 & 2 & 3 & 4 & 5 & 6 & 7 & 8 & 9 \\
\hline
\end{tabular}

11. Imaginez que vous vous piquez le bout du doigt avec une épine de rose.

$0=$ Aucune douleur

La plus forte douleur imaginable $=10$

\begin{tabular}{|l|l|l|l|l|l|l|l|l|l|l|}
\hline 0 & 1 & 2 & 3 & 4 & 5 & 6 & 7 & 8 & 9 & 10 \\
\hline
\end{tabular}

12. Imaginez que vous mettez vos mains nues dans la neige pendant quelques minutes, ou que vous restez en contact avec de la neige pendant un moment, par exemple en faisant des boules de neiges.

$0=$ Aucune douleur $\quad$ La plus forte douleur imaginable $=10$

\begin{tabular}{|l|l|l|l|l|l|l|l|l|l|l|}
\hline 0 & 1 & 2 & 3 & 4 & 5 & 6 & 7 & 8 & 9 & 10 \\
\hline
\end{tabular}

13. Imaginez que vous serrez la main de quelqu'un qui aurait une poignée de main normale.

$0=$ Aucune douleur

La plus forte douleur imaginable $=10$

\begin{tabular}{|l|l|l|l|l|l|l|l|l|l|l|}
\hline 0 & 1 & 2 & 3 & 4 & 5 & 6 & 7 & 8 & 9 & 10 \\
\hline
\end{tabular}

14. Imaginez que vous serrez la main de quelqu'un qui aurait une poignée de main très forte.

$0=$ Aucune douleur

La plus forte douleur imaginable $=10$

\begin{tabular}{|l|l|l|l|l|l|l|l|l|l|}
\hline 0 & 1 & 2 & 3 & 4 & 5 & 7 & 9 & 10
\end{tabular}

15. Imaginez que vous attrapez sans le faire exprès, une cocotte brûlante par ses poignées, qui seraient aussi brûlantes.

$0=$ Aucune douleur

La plus forte douleur imaginable $=10$

\begin{tabular}{|l|l|l|l|l|l|l|l|l|l|l|}
\hline 0 & 1 & 2 & 3 & 4 & 5 & 6 & 7 & 8 & 9 & 10 \\
\hline
\end{tabular}

16. Imaginez que vous portez des sandales et que quelqu'un vous marche sur le pied avec une grosse chaussure.

$0=$ Aucune douleur

La plus forte douleur imaginable $=10$

\begin{tabular}{l|l|l|l|l|l|l|l|l|l|l|l}
0 & 1 & 2 & 3 & 4 & 5 & 6 & 7 & 8 & 9 & 10
\end{tabular}

17. Imaginez que vous vous cognez le nerf derrière le coude sur le bord de la table. $0=$ Aucune douleur La plus forte douleur imaginable $=10$

\begin{tabular}{|l|l|l|l|l|l|l|l|l|l|}
\hline 0 & 1 & 2 & 3 & 4 & 5 & 6 & 7 & 8 & 9 \\
\hline
\end{tabular}




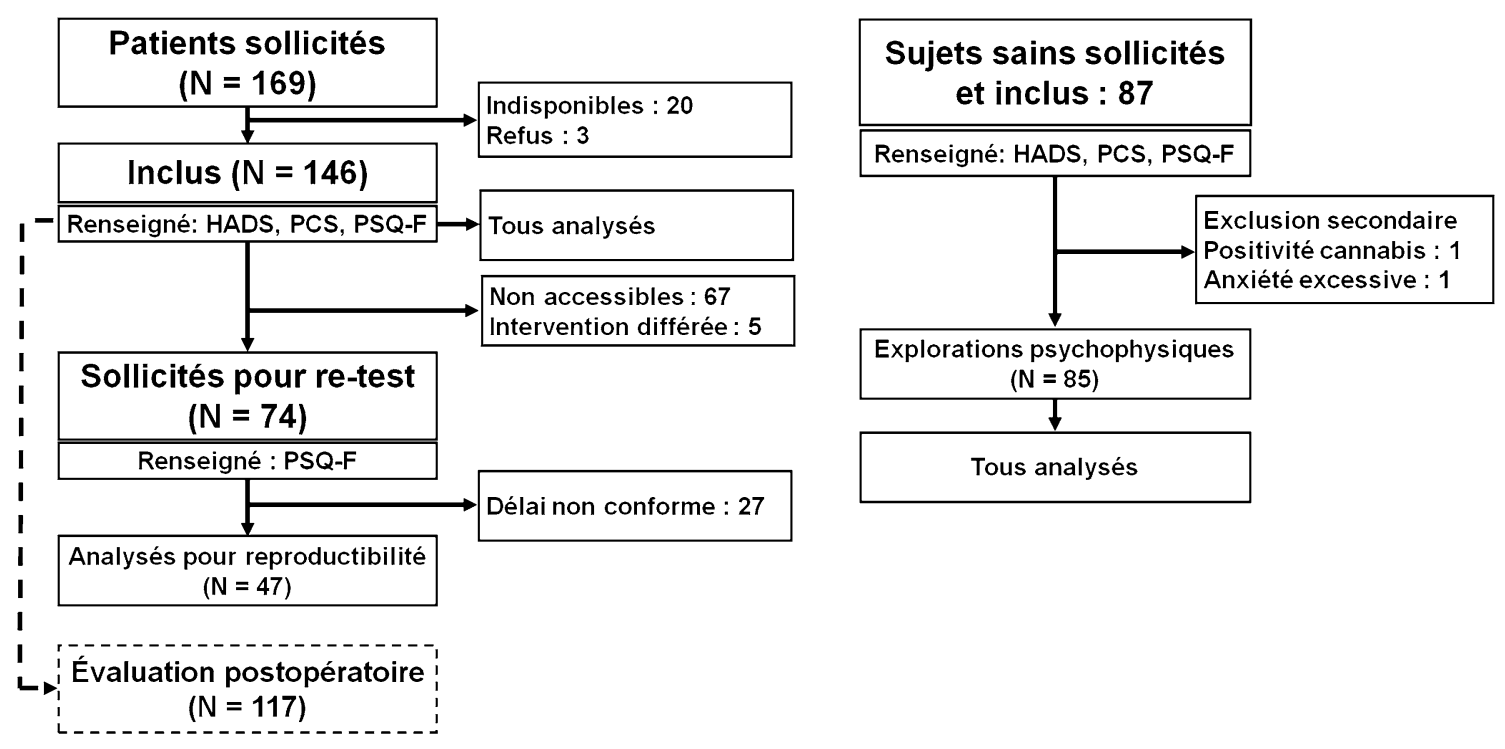

Figure 1 Diagramme de flux

Tableau 2 Description de l'échantillon d'étude

$\begin{array}{ll}\begin{array}{l}\text { Patients } \\ (n=146)\end{array} & \text { Sujets sain } \\ (n=85)\end{array}$

Démographie / morphométrie

Sexe féminin

$94(64,4)$

$43(50,6)$

Age (années)

$45,9(13,5)$

$32,2(11,3)$

IMC $\left(\mathrm{kg} \cdot \mathrm{m}^{-2}\right)$

$26,8(5,7)$

Questionnaire HADS

Score d'anxiété

$8,4(4,1)$

$4,5(2,4)$

Score d'anxiété $>8$

$56(38,6)$

$5(5,9)$

Score de dépression

$4,2(3,4)$

$1,5(1,6)$

Score de dépression $>8$

$15(10,3)$

$0(0,0)$

Questionnaire PCS

Score de rumination

$4,6(3,5)$

Score d'exagération

$2,0(1,7)$

Score d'impuissance

Score de catastrophisme

$17,9(13,2)$

$11,6(8,3)$

Les variables numériques sont exprimées en moyenne (écart-type); les variables catégorielles sont exprimées en nombre (pourcentage). $H A D S=$ Hospital Anxiety \& Depression Scale; IMC = indice de masse corporelle; PCS = Pain Catastrophizing Scale. Pour le HADS, des scores supérieurs à 8 font suspecter l'existence d'un trouble anxieux ou dépressif, dont la certitude est signalée par des scores supérieurs à 11 (Zigmond AS, Snaith RP. Acta Psychiatr Scand 1983; 67: 361-70). Il n'y a pas de valeur-seuil pour le PCS, dont les valeurs maximales théoriques sont respectivement 16, 12, 24 et 52 pour les scores de rumination, exagération, impuissance et catastrophisme (somme des trois premiers) (Sullivan MJ, Bishop SR, Pivik J. Psychol Assess 1995; 7: 524-32). IMC = indice de masse corporelle

\section{Validité convergente}

Le Tableau 5 montre les relations entre le PSQ-F, et les échelles HADS et PCS. Elles différaient entre les patients (corrélation positive pour tous les facteurs testés) et les sujets sains (aucune corrélation significative, avec des coefficients pour la plupart négatifs et très proches de 0 ).
Validité de structure contre critère externe

Chez 117 patients $(80,1 \%)$, un score de satisfaction vis-à-vis de la qualité de l'analgésie a pu être relevé, sa médiane étant de $9 / 10(8-10) ; 83,8 \%$ des répondants rapportaient une satisfaction $>7 / 10$. Cette distorsion vers 
Tableau 3 Validité de structure interne et reproductibilité

\begin{tabular}{|c|c|c|c|c|c|c|c|}
\hline \multirow[t]{3}{*}{ Item } & \multicolumn{4}{|c|}{ Patients $(n=146)$} & \multicolumn{3}{|c|}{ Sujets sains $(n=85)$} \\
\hline & \multicolumn{2}{|c|}{ Analyse factorielle } & \multicolumn{2}{|l|}{ Corrélation } & \multicolumn{2}{|c|}{ Analyse factorielle } & \multirow{2}{*}{$\begin{array}{l}\text { Corrélation avec } \\
\text { score total * }\end{array}$} \\
\hline & Facteur 1 & Facteur 2 & $\begin{array}{l}\text { avec score } \\
\text { total } *\end{array}$ & $\begin{array}{l}\text { intra-classe } \\
\text { (test-retest) }\end{array}$ & Facteur 1 & Facteur 2 & \\
\hline$n^{\circ} 1$ & 0,719 & 0,313 & 0,685 & 0,667 & 0,751 & $-0,017$ & 0,480 \\
\hline$n^{\circ} 2$ & 0,736 & 0,272 & 0,670 & 0,565 & 0,642 & 0,257 & 0,593 \\
\hline$n^{\circ} 3$ & 0,711 & 0,217 & 0,609 & 0,605 & 0,359 & 0,338 & 0,448 \\
\hline$n^{\circ} 4$ & 0,806 & 0,221 & 0,684 & 0,554 & $\mathbf{0 , 8 2 1}$ & 0,090 & 0,592 \\
\hline$n^{\circ} 6$ & 0,270 & $\mathbf{0 , 5 5 2}$ & 0,516 & 0,617 & $-0,078$ & 0,790 & 0,427 \\
\hline$n^{\circ} 7$ & $\mathbf{0 , 5 3 1}$ & 0,515 & 0,689 & 0,454 & 0,347 & 0,624 & 0,615 \\
\hline$n^{\circ} 8$ & 0,638 & 0,446 & 0,722 & 0,661 & 0,609 & $\mathbf{0 , 5 3 2}$ & 0,743 \\
\hline $\mathrm{n}^{\circ} 10$ & 0,541 & $\mathbf{0 , 5 3 5}$ & 0,707 & 0,338 & 0,419 & 0,696 & 0,721 \\
\hline $\mathrm{n}^{\circ} 11$ & 0,404 & 0,733 & 0,751 & 0,540 & 0,463 & 0,739 & 0,791 \\
\hline $\mathrm{n}^{\circ} 12$ & 0,047 & 0,842 & 0,553 & 0,597 & 0,336 & 0,641 & 0,624 \\
\hline $\mathrm{n}^{\circ} 14$ & 0,325 & $\mathbf{0 , 7 5 8}$ & 0,709 & 0,787 & 0,107 & 0,770 & 0,532 \\
\hline $\mathrm{n}^{\circ} 15$ & 0,686 & 0,220 & 0,586 & 0,688 & 0,682 & 0,373 & 0,683 \\
\hline $\mathrm{n}^{\circ} 16$ & 0,353 & 0,698 & 0,681 & 0,543 & 0,633 & 0,348 & 0,624 \\
\hline $\mathrm{n}^{\circ} 17$ & 0,495 & 0,600 & 0,724 & 0,671 & $\mathbf{0 , 7 1 7}$ & 0,284 & 0,636 \\
\hline
\end{tabular}

Analyse des 14 items représentatifs de la sensibilité à la douleur, chez les patients (à gauche) et les sujets sains (à droite). Pour l'analyse factorielle sont représentés les poids de chaque item pour chacun des deux premiers facteurs retenus (F1 et F2). Chez les patients, F1 et F2 représentaient respectivement 51,7 et 8,4\% de la variance totale. Chez les volontaires sains, F1 et F2 représentaient respectivement 46,1 et 11,3 $\%$ de la variance totale. Le poids de chaque item dans l'analyse factorielle est signalé en gras lorsqu'il dépasse la valeur critique de 0,4 . Les coefficients de corrélation sont le $\mathrm{r}$ de Pearson pour la corrélation avec le score total et le coefficient intra-classe pour le test-retest (patients uniquement)

*Corrélation corrigée après retrait de l'item

Tableau 4 Scores du PSQ-F observés

\begin{tabular}{lll}
\hline & $\begin{array}{l}\text { Patients } \\
(n=146)\end{array}$ & $\begin{array}{l}\text { Sujets sains } \\
(n=85)\end{array}$ \\
\hline Sous-score «mineur » & $2,7(1,5)$ & $3,0(1,3)$ \\
Sous-score «modéré » & $4,9(1,6)$ & $5,2(1,3)$ \\
Score total & $3,8(1,5)$ & $4,1(1,2)$ \\
\hline
\end{tabular}

Valeurs des scores issus de la version française du Pain Sensitivity Questionnaire (PSQ-F) dans les deux groupes de l'étude. Les variables numériques sont exprimées en moyenne (écart-type)

PSQ-F = version française du Pain Sensitivity Questionnaire

le haut reflétant une analgésie très efficace, il n'a pas été fait d'étude de corrélation avec le PSQ-F.

Le Tableau 6 montre les résultats des stimulations nociceptives chez les sujets sains, et leur relation avec le PSQ-F. On note une corrélation négative entre les trois scores du PSQ-F et le T\%/douleur-6, et une corrélation positive entre les scores mineur et total du PSQ-F, et la moyenne des 30 premières secondes de la douleur au CPT.

\section{Discussion}

Cette étude a donné des résultats satisfaisants en termes de validité interne, de reproductibilité et de validité externe. Toutefois, nous devons discuter des différences observées entre nos résultats et ceux des études antérieures réalisées avec la version originale et des versions traduites.

La consistance interne que nous avons retrouvée chez les patients est très proche de celle observée chez des sujets sains, dans les études originale, coréenne et chinoise. ${ }^{9,12,14}$ La reproductibilité était en revanche meilleure chez les 
Tableau 5 Validité convergente

\section{PSQ-F}

Sous-score « mineur »

Sous-score «modéré »

Score total

Patients $(n=146)$

Questionnaire HADS

Score d'anxiété

Score de dépression

Questionnaire PCS

Score de rumination

0,344

0,304

$\mathbf{0 , 3 3 9}$

Score d'exagération

0,344

0,217

0,284

$\mathbf{0 , 4 1 7}$

0,341

0,391

0,404

$\mathbf{0 , 3 2 9}$

0,380

Sujets sains $(\boldsymbol{n}=\mathbf{8 5})$

Questionnaire HADS

\section{Score d'anxiété}

$-0,144$

$-0,084$

$-0,132$

Score de dépression

$-0,078$

Questionnaire PCS

Score de rumination

$-0,073$

$-0,040$

$-0,076$

Score d'exagération

0,048

0,177

0,108

Score d'impuissance

0,055

0,005

Score de catastrophisme

0,023

$-0,030$

Pour chaque relation est donné le coefficient $\rho$ de corrélation de Spearman, les valeurs notées en gras correspondant à une différence significative avec la valeur nulle $(P<0,05$; inflation du risque $\alpha$ non corrigée). HADS $=$ Hospital Anxiety \& Depression Scale; PCS = Pain Catastrophizing Scale; PSQ-F = version française du Pain Sensitivity Questionnaire

Tableau 6 Validité de structure contre critère externe

\begin{tabular}{|c|c|c|c|c|}
\hline \multirow[t]{2}{*}{ Variable } & \multirow[t]{2}{*}{ Valeur } & \multicolumn{3}{|c|}{ Corrélation au PSQ-F } \\
\hline & & $\begin{array}{l}\text { Sous-score } \\
« \text { mineur } »\end{array}$ & $\begin{array}{l}\text { Sous-score } \\
\text { «modéré » }\end{array}$ & $\begin{array}{l}\text { Score } \\
\text { total }\end{array}$ \\
\hline \multicolumn{5}{|l|}{ Von Frey électronique (sensibilité mécanique ponctuelle) } \\
\hline Seuil de nociception $(\mathrm{g})$ & $188(134-265)$ & $-0,051$ & $-0,032$ & $-0,031$ \\
\hline \multicolumn{5}{|l|}{ Thermique chaud (Pathway®) } \\
\hline Seuil de sensibilité $\left({ }^{\circ} \mathrm{C}\right)$ & $\begin{array}{l}35,1(34,3- \\
36,5)\end{array}$ & $\mathrm{n} / \mathrm{c}$ & & \\
\hline Seuil de nociception $\left({ }^{\circ} \mathrm{C}\right)$ & $\begin{array}{l}43,6(40,9- \\
\quad 45,6)\end{array}$ & $-0,147$ & $-0,113$ & $-0,129$ \\
\hline $\mathrm{T}^{\circ} /$ douleur-6 $\left({ }^{\circ} \mathrm{C}\right)$ & $47(44,4-48,2)$ & $-0,390$ & $-0,376$ & $-0,426$ \\
\hline \multicolumn{5}{|l|}{ Cold pressure test } \\
\hline Durée d'immersion & $41(23-120)$ & $-0,060$ & 0,000 & $-0,024$ \\
\hline $\begin{array}{l}\text { Douleur moyenne ressentie (moyenne des } 30 \text { premières secondes) } \\
\text { (EVA sur 10) }\end{array}$ & $5,4(2,5-6,9)$ & 0,241 & 0,141 & 0,218 \\
\hline Douleur moyenne ressentie (moyenne des 2 minutes) (EVA sur 10) & $8,6(6,6-9,1)$ & 0,177 & 0,055 & 0,128 \\
\hline
\end{tabular}

Résultats des tests psychophysiques réalisés chez les sujets sains $(n=85)$ et validité de structure contre critère externe des scores issus de la version française du Pain Sensitivity Questionnaire (PSQ-F). Les variables numériques sont exprimées en médiane (écart inter-quartile). Pour chaque relation entre variables numériques est donné le coefficient $\rho$ de corrélation de Spearman, les valeurs notées en gras correspondant à une différence significative avec la valeur nulle $(P<0,05$; inflation du risque $\alpha$ non corrigée). EVA $=$ échelle visuelle analogique; n/c $=$ non calculé 
sujets sains des études originale et chinoise, ${ }^{9,14}$ que chez nos patients en attente d'une chirurgie ou d'un accouchement. Le contexte clinique a donc pu altérer la reproductibilité, fait également constaté chez des patients atteints de lombarthrose douloureuse. ${ }^{12,13}$

En termes de validité convergente, l'étude originale sur sujets sains rapporte une corrélation des trois scores du PSQ avec le catastrophisme, ainsi que du score mineur avec l'état anxieux et la dépression. ${ }^{9}$ L'étude chinoise, dont l'échantillon comportait un taux élevé de personnes jeunes et éduquées, retrouvait une corrélation avec le PCS, quoique plus faible, une corrélation faible et inconstante avec l'anxiété, et pas de corrélation avec la dépression. ${ }^{14}$ L'absence de relation chez nos sujets sains peut être due à un recrutement qui a évité - à la demande du comité d'éthique - les troubles psychologiques préexistants, d'où des scores psychométriques bas et peu variables. Aussi, le catastrophisme dépendant de l'expérience des douleurs ressenties dans le passé, ${ }^{25}$ le recrutement de sujets plus âgés a pu altérer la relation avec le PSQ-F. Chez les patients en revanche, où nous retrouvons une corrélation du PSQ-F avec anxiété, dépression et catastrophisme, nos résultats sont cohérents avec les résultats publiés. Pour la validation anglophone chez 136 patients douloureux chroniques avant infiltration lombaire, ${ }^{11}$ les trois scores du PSQ étaient corrélés au catastrophisme, mais pas à l'anxiété ni à la dépression. Pour la validation coréenne chez 72 patients atteints de lombarthrose douloureuse, la relation avec le catastrophisme était similaire. ${ }^{12}$ Sinon, une équipe suisse a testé une traduction française du PSQ (sans décrire le contenu ni les étapes de validation interne), chez 198 patientes avant chirurgie du cancer du sein. ${ }^{26}$ Le PSQ total était corrélé au trait (mais pas à l'état) anxieux, à la dépression, et à l'intensité de douleur attendue. Dans l'ensemble, chez des patients plus exposés à des troubles psychologiques, et plus encore en cas de douleur chronique, la validité convergente du PSQ est meilleure.

En termes de validité externe, nous retrouvons, avec des effets plus faibles et une significativité moins constante, les tendances observées notamment dans l'étude originale qui retrouvait une relation du PSQ avec la douleur induite, mais pas avec les seuils nociceptifs, ${ }^{9}$ et dans l'étude chinoise, avec la douleur induite et les seuils nociceptifs, mais pas de tolérance. ${ }^{14}$ Nous n'avons pas testé la validité externe chez les patients, comme fait avant infiltration lombaire ou mammaire, où le PSQ-total est corrélé à la douleur expérimentale. ${ }^{11,26}$ En situation de douleur chronique, la corrélation a même été retrouvée avec les seuils nociceptifs. ${ }^{27}$ Enfin, le PSQ-total prédirait la douleur postopératoire après chirurgie mammaire. ${ }^{26} \mathrm{~A}$ ce propos, il sera essentiel de réaliser ces études prédictives dans chaque type de chirurgie - au moins par grandes catégories - afin de pouvoir valider l'utilité du PSQ dans ce contexte.
N'ayant pas cet objectif, notre étude, en ciblant des types de chirurgie variés, au risque d'avoir des sous-groupes de petite taille, ne permettait pas une telle approche.

Ce PSQ-F est donc valide, et utilisable d'ores et déjà par les cliniciens. On notera toutefois que la relation du PSQ avec les autres dimensions psycho-cliniques tend à augmenter avec l'expérience douloureuse du sujet. De fait, s'il est testé comme prédicteur d'une douleur postopératoire, le modèle prédictif ne serait valide que pour les types de chirurgie testés. De plus, l'utilité respective du score total du PSQ et de ses deux sous-scores reste à déterminer, notamment leur faculté prédictive d'un évènement douloureux pertinent cliniquement. On ajoutera qu'en termes d'utilité pour la recherche, le sous-score mineur a été utilisé comme « version courte » en épidémiologie. ${ }^{28}$

Remerciements Alain Artola (INSERM, UMR1107), Sandra Guerrin et Sandra Guichard pour les traductions Allemand/Français; Cornelia Schulze pour la rétro-traduction; Isabelle Genès pour l'implication de l'école d'IADE de Clermont-Ferrand dans le test de la version pilote et la collecte des données; Claire Amiens, Jennifer Blanchard, Ophélie Gautier, Mickaël Niçoise, et Angélique Rodat pour la collecte des données; Ghislaine Niezgodzki et Sylvie Sonneville pour le contrôle de la qualité des données; Sylvia Boulliau, Fatiha Giron, Nicolas Macian et Vincent Leray pour les épreuves psychophysiques.

Conflit d'intérêt Aucun déclaré.

Responsabilité éditoriale Cet article a été traité par Dr Étienne de Médicis, rédacteur de la langue française, Journal canadien d'anesthésie.

Contributions des auteurs Christian Dualé a conçu et dirigé l'étude, interprété les résultats et rédigé le manuscrit. Candy GuiguetAuclair a conçu l'étude, analysé et interprété les résultats, et participé à la rédaction du manuscrit. Ruth Ruscheweyh et Claude Dubray ont participé à la conception de l'étude et à la rédaction du manuscrit. Ulrich Bauer, Vedat Eljezi, Sylvie Eschalier et Brigitte Storme ont réalisé l'investigation et la collection des données.

Financement L'étude n'a pas bénéficié de financement extérieur.

\section{Références}

1. Denk F, McMahon SB, Tracey I. Pain vulnerability: a neurobiological perspective. Nat Neurosci 2014; 17: 192-200.

2. Denk F, McMahon SB. Neurobiological basis for pain vulnerability: why me? Pain 2017; 158(Suppl 1): S108-14.

3. Dualé C, Nicolas-Courbon A, Gerbaud L, Lemery D, Bonnin M, Pereira B. Maternal satisfaction as an outcome criterion in research on labor analgesia: data analysis from the recent literature. Clin J Pain 2015; 31: 235-46.

4. Abrishami A, Chan J, Chung F, Wong J. Preoperative pain sensitivity and its correlation with postoperative pain and 
analgesic consumption: a qualitative systematic review. Anesthesiology 2011; 114: 445-57.

5. Rago $R$, Forfori F, Materazzi $G$, et al. Evaluation of a preoperative pain score in response to pressure as a marker of postoperative pain and drugs consumption in surgical thyroidectomy. Clin J Pain 2012; 28: 382-6.

6. Persson AK, Pettersson FD, Dyrehag LE, Akeson J. Prediction of postoperative pain from assessment of pain induced by venous cannulation and propofol infusion. Acta Anaesthesiol Scand 2016; 60: 166-76.

7. Petersen $K K$, Graven-Nielsen $T$, Simonsen $O$, Laursen $M B$, Arendt-Nielsen L. Preoperative pain mechanisms assessed by cuff algometry are associated with chronic postoperative pain relief after total knee replacement. Pain 2016; 157: 1400-6.

8. Carvalho B, Zheng $M$, Aiono-LeTagaloa L. Evaluation of experimental pain tests to predict labour pain and epidural analgesic consumption. Br J Anaesth 2013; 110: 600-6.

9. Ruscheweyh R, Marziniak M, Stumpenhorst F, Reinholz J, Knecht $S$. Pain sensitivity can be assessed by self-rating: development and validation of the Pain Sensitivity Questionnaire. Pain 2009; 146: $65-74$

10. Kim HJ, Lee JI, Kang KT, et al. Influence of pain sensitivity on surgical outcomes after lumbar spine surgery in patients with lumbar spinal stenosis. Spine (Phila Pa 1976) 2015; 40: 193200.

11. Sellers AB, Ruscheweyh R, Kelley BJ, Ness TJ, Vetter TR. Validation of the English language pain sensitivity questionnaire. Reg Anesth Pain Med 2013; 38: 508-14.

12. Kim HJ, Ruscheweyh R, Yeo JH, et al. Translation, cross-cultural adaptation, and validity of the Korean version of the pain sensitivity questionnaire in chronic pain patients. Pain Pract 2014; 14: 745-51.

13. Azimi $P$, Azhari $S$, Shahzadi $S$, et al. Outcome measure of pain in patients with lumbar disc herniation: validation study of the Iranian version of Pain Sensitivity Questionnaire. Asian Spine J 2016; 10: 480-7.

14. Quan X, Fong DY, Leung AY, Liao Q, Ruscheweyh R, Chau PH. Validation of the Mandarin Chinese version of the pain sensitivity questionnaire. Pain Pract 2018; 18: 180-93.

15. Vallerand $R J$. Vers une méthodologie de validation transculturelle de questionnaires psychologiques : implications pour la recherche en langue française. Can Psychol 1989; 30: 662-80.
16. Guillemin F, Bombardier C, Beaton D. Cross-cultural adaptation of health-related quality of life measures: literature review and proposed guidelines. J Clin Epidemiol 1993; 46: 1417-32.

17. Beaton DE, Bombardier C, Guillemin F, Ferraz MB. Guidelines for the process of cross-cultural adaptation of self-report measures. Spine (Phila Pa 1976) 2000; 25: 3186-91.

18. Sullivan MJ, Bishop SR, Pivik J. The pain catastrophizing scale: development and validation. Psychol Assess 1995; 7: 524-32.

19. French DJ, Noël M, Vigneau F, et al. L'échelle de dramatisation face à la douleur PCS-CF : adaptation canadienne en langue française de l'échelle «Pain Catastrophizing Scale». Can J Behav Sci 2005; 37: 181-92.

20. Zigmond AS, Snaith RP. The hospital anxiety and depression scale. Acta Psychiatr Scand 1983; 67: 361-70.

21. Lépine JP, Godchau M, Brun P, Lempirière P. Evaluation de l'anxiété et de la dépression chez des patients hospitalisés dans un service de médecine interne. Ann Med Psychol (Paris) 1985; 143: 175-89.

22. Feise RJ. Do multiple outcome measures require p-value adjustment? BMC Med Res Methodol 2002; 2: 8.

23. Perneger TV. What's wrong with Bonferroni adjustments. BMJ 1998; 316: 1236-8.

24. Terwee $C B$, Bot $S D$, de Boer $M R$, et al. Quality criteria were proposed for measurement properties of health status questionnaires. J Clin Epidemiol 2007; 60: 34-42.

25. Ruscheweyh $R$, Nees $F$, Marziniak $M$, Evers $S$, Flor $H$, Knecht $S$. Pain catastrophizing and pain-related emotions: influence of age and type of pain. Clin J Pain 2011; 27: 578-86.

26. Rehberg B, Mathivon S, Combescure C, Mercier Y, Savoldelli $G L$. Prediction of acute postoperative pain following breast cancer surgery using the pain sensitivity questionnaire: a cohort study. Clin J Pain 2017; 33: 57-66.

27. Ruscheweyh $R$, Verneuer B, Dany $K$, et al. Validation of the pain sensitivity questionnaire in chronic pain patients. Pain 2012; 153 : 1210-8.

28. Hermesdorf M, Berger K, Baune BT, Wellmann J, Ruscheweyh $R$, Wersching $H$. Pain sensitivity in patients with major depression: differential effect of pain sensitivity measures, somatic cofactors, and disease characteristics. J Pain 2016; 17: 606-16.

Publisher's Note Springer Nature remains neutral with regard to jurisdictional claims in published maps and institutional affiliations. 